\title{
Ovarian Dermoid Cyst with Melanoma
}

National Cancer Institute

\section{Source}

National Cancer Institute. Ovarian Dermoid Cyst with Melanoma. NCI Thesaurus. Code C40001.

A secondary melanoma that has developed in an ovarian dermoid cyst. 